\title{
Ambientes Virtuais de Aprendizagem em Interfaces Gestuais: potencialidades para Pessoas com Dispraxia
}

Recibido: 1 Julio 2015 - Revisado: 30 Septiembre 2015

Aceptado: 30 Octubre 2015 - Publicado: 30 Diciembre 2015

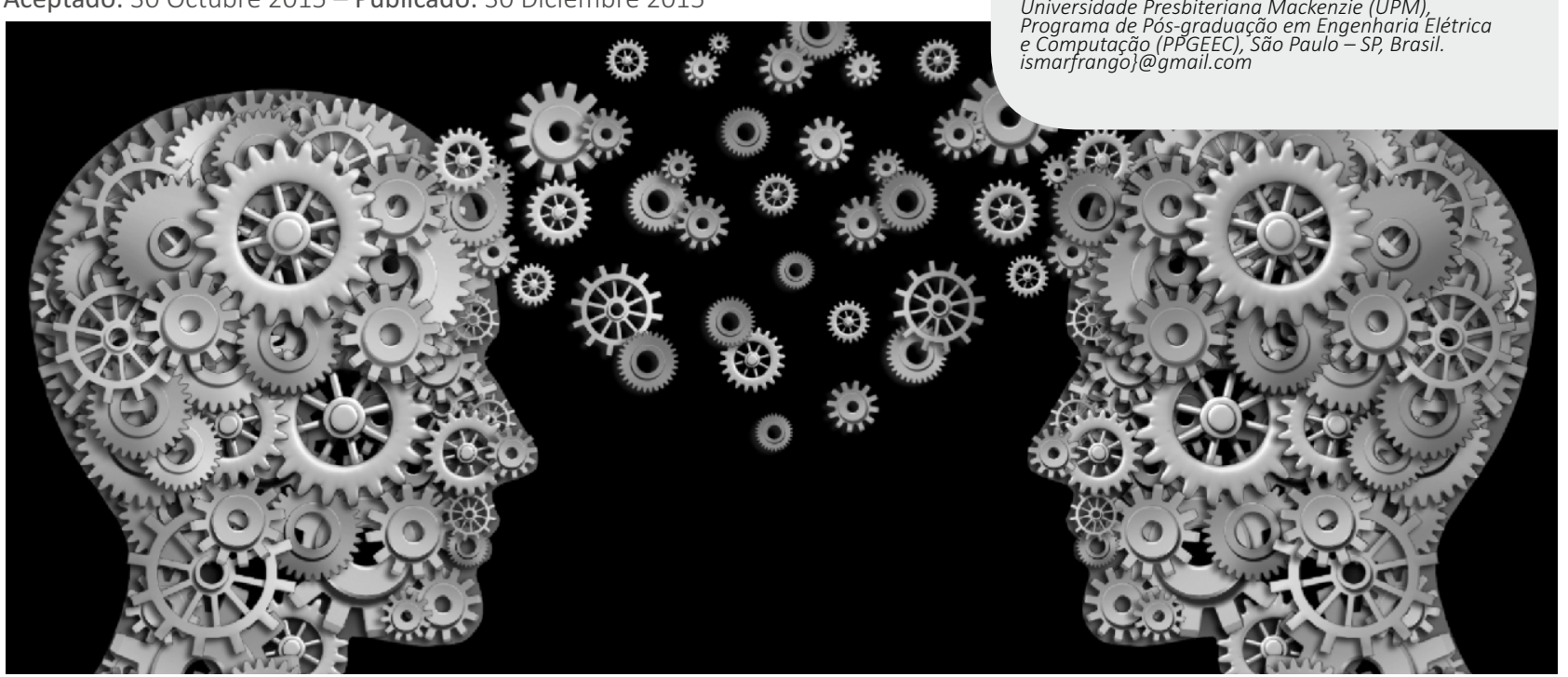

Resumo: Este trabalho discute os potenciais que os Ambientes Virtuais de Aprendizagem podem trazer para Pessoas com Dispraxia ao serem acessados através de interfaces gestuais, que são meios de acesso mais intuitivos e naturais às suas realidades. As tecnologias touchless podem diminuir as dificuldades de aprendizagem, letramento, inclusão digital, educacional e social destes sujeitos. Recomenda-se a adaptação do Moodle para ser utilizado com o Leapmotion por dispráxicos, envolvendo estudos preliminares e a participação de profissionais e usuários finais. Como resultado desta fase de projeto, espera-se criar guidelines que norteiem como devem ser realizadas as adaptações na arquitetura do Moodle para mudar o módulo de interface e que, nas etapas seguintes, as mudanças sejam testadas por mais pessoas com TDC para análise de comportamento durante a interação e melhoria dessas diretrizes.

Palavras-Chave Interfaces Gestuais; Ambientes Virtuais de Aprendizagem; Dispraxia; Transtorno do Desenvolvimento da Coordenação.

Abstract: This work discusses the potential that the Virtual Learning Environments can to bring for people with Dyspraxia using gestural interfaces that are more intuitive and natural. The touchless technologies can reduce the difficulties of learning, literacy, the digital, educational and social inclusion of these users. We recommend the Moodle's adaptation for to be used with the Leapmotion by dyspraxics, involving preliminary studies and the participation of the professionals and final users. As a result of this project, we hope to create guidelines about how to do the adaptations in the Moodle's architectures and to change the interface module. And the other phase, we will test the changes in more people with TDC for behavior analysis during the interaction and improving these guidelines.

Index Terms Gestural Interfaces; Virtual Learning Environments; Dyspraxia; Developmental Coordination Disorder. 


\section{INTRODUÇÃO}

Um Ambiente Virtual de Aprendizagem (AVA) deve ter uma interface amigável e intuitiva para disponibilizar conteúdos e ser utilizada no ensino a distância, proporcionando interação e cooperação entre os usuários, tendo como exemplo o Moodle.

Estes ambientes de ensino devem ser acessíveis e disponíveis para uma educação ao alcance de todos, incluindo a integração com tecnologias que se utilizam de interfaces gestuais para pessoas com Dispraxia, já que este é um distúrbio que compromete a coordenação motora, influenciando na utilização correta de ferramentas digitais.

Desta forma, ambientes virtuais de ensino aprendizagem podem ser utilizados através das interfaces gestuais por dispráxicos em seus processos de inclusão digital, educacional e social, sendo um potencial de melhoria na qualidade de vida desses sujeitos, necessitando-se de adaptações na arquitetura do AVA e seguindo diretrizes de como essas adequações devem ser realizadas.

O artigo encontra-se assim organizado: a Seção 2 faz a fundamentação teórica e metodológica sobre ambientes virtuais de aprendizagem, Dispraxia e interfaces gestuais, abordando-se as características destes conceitos, a justificativa e a parte metodológica. Em seguida, na Seção 3, apresenta-se o panorama geral da pesquisa destacando a proposta de estudo e os trabalhos já existentes. Por fim, na Seção 4 são colocadas as considerações finais e etapas seguintes desta investigação.

\section{FUNDAMENTAÇÃO TEÓRICA E METODOLÓGICA}

Ambientes Virtuais de Aprendizagem (AVAs) são sistemas para aprendizagem com interface amigável e intuitiva, normalmente utilizados no ensino a distância, disponibilizando materiais de forma organizada, além de proporcionar interação e trabalho através de ferramentas de comunicação e cooperativamente entre os usuários [da Costa Ferro et al. 2014].

O Moodle (Modular Object Oriented Distance Learning) é um AVA de código aberto e estável, desenvolvido em PHP e projetado em princípios pedagógicos para oferecer recursos educacionais, dando a possibilidade de configuração e gerenciamento de atividades [da Silva et al. 2014]. Além disso, é desenvolvido em módulos e sua arquitetura é focada no ambiente de um curso funcionalidades de acordo com as necessidades.

Os AVAs, de modo geral, devem ser acessíveis e disponíveis para uma educação ao alcance de todos de acordo com os princípios de Design Universal [Nielsen et al. 2004], proporcionando uma potencialidade na melhoria da qualidade de vida, inclusão educacional, digital e social [Cardoso et al. 2014], ampliação da comunicação, igualdade de condições, mobilidade e desenvolvimento de habilidades [Paola-J et al. 2014].

Um exemplo de distúrbio em que a educação a distância e os AVAs podem influenciar na utilização correta de tecnologias digitais e ajudar na escolarização e letramento dos sujeitos é a Dispraxia. É também conhecida como Transtorno do Desenvolvimento da Coordenação (TDC), sendo caracterizada principalmente por dificuldades espacial, motora, postural e verbal, comprometendo os movimentos, percepções, pensamentos e a linguagem [Portwood 2013].

O TDC é um distúrbio ligado à coordenação motora que compromete no rendimento escolar, a educação física e nas atividades cotidianas como se vestir, higiene pessoal, alimentação, relações sociais e saúde. Não há lesão no cérebro clinicamente evidente, já que este é impedido de desempenhar todas suas funções. Compromete o equilíbrio, gerando imprecisão e lentidão, pois é uma disfunção motora neurológica [Polatajko and Cantin 2005]. 
Os dispráxicos têm problemas em relação aos conceitos de alto, baixo, longe, perto, com formas e tamanhos de figuras. Seus movimentos são sem ritmo e com pouco controle, comprometendo a postura. Em alguns casos, a linguagem não é afetada, mas existe déficit fonológico e fonético na fala.

Tecnologias que se utilizam de interfaces gestuais também podem ajudar pessoas com Dispraxia, pois podem diminuir as dificuldades inerentes ao transtorno e aumentar suas habilidades na medida em que promovem uma interação humano-computador mais flexível que outras. Elas reforçam a aprendizagem, comunicação e memorização de ações digitais através de movimentos e gestos cinestésicos realizados no ar [Garzotto et al. 2014].

As interfaces gestuais ou touchless são sistemas computacionais em que a comunicação ocorre sem contato. Há uma relação sem interação física ou mecânica de dispositivos periféricos, como o mouse, teclado, voz ou toque pela superfície da tela (touchscreen) [Placitelli and Gallo 2012]. É um processo interativo mais natural através de interfaces, que reconhecem gestos, expressões ou partes do corpo humano, como mãos, olhos, voz, pulsos elétricos emitidos pelo cérebro, músculos ou nervos, podendo ocorrer também a combinação de várias partes [Erazo and Pino 2014].

Ao se utilizar as mãos como forma de reconhecimento dos gestos por essas interfaces, por exemplo, necessita-se do mapeamento das orientações e posições para que um sistema digital identifique quais tarefas devem ser executadas. Para isso, empregamse softwares e dispositivos de hardware como antenas, bastões, braceletes, câmeras, luvas, microfones e sensores de postura, presença ou profundidade [Nielsen et al. 2004].

As interfaces gestuais devem obedecer também aos princípios da usabilidade da Interface HumanoComputador (IHC) e de desenho das interfaces
[Norman and Nielsen 2010], provendo uma educação a distancia ao alcance de todos. E para se comunicarem efetivamente com os AVAs, necessitase de adaptações em suas arquiteturas e de diretrizes ou guidelines que norteiem como devem ser realizadas.

Como exemplos de aplicativos existentes que trabalham com interfaces touchless, podem-se citar: ASUS Xtion Motion Sensor, GestureTek - Projeções Interativas (Parede, Piso), Leapmotion, Microsoft Kinect (Xbox 360)- Win And I, MYO Armband (Thalmic Labs), Nintendo Wii (U), PlayStation Move e Eye (Sony) e Wisee: Wi-Fi signals, dentre outros.

Como justificativa para a pesquisa, portanto, tem-se que as tecnologias de interfaces gestuais podem ajudar pessoas com Dispraxia, de forma que, integrando-as particularmente ao Moodle, possam diminuir as dificuldades de aprendizagem, letramento, inclusão digital, educacional e social, melhorando também as habilidades de comunicação através da utilização de ferramentas tecnológicas.

A metodologia utilizada será através de estudos bibliográficos pela análise de documentos contemporâneos e cientificamente relevantes, em que tenham ocorrido adequações na arquitetura do Moodle e criação de guidelines para esse tipo de adaptações.

Para isso, entretanto, questões práticas e éticas devem ser envolvidas em um trabalho de integração entre o Moodle e as interfaces gestuais, pois, na criação de diretrizes para adaptação deste AVA com uma tecnologia touchless para dispráxicos, deve haver a participação de diferentes profissionais e levar em consideração sujeitos com esse transtorno, suas opiniões, necessidades e limitações, além de obedecer aos princípios de Design Universal [Antonio et al. 2014]. 


\section{PROPOSTA DE INVESTIGAÇÃO}

Este estudo propõe uma discussão teórica sobre a adaptação de Ambientes Virtuais de Aprendizagem para as tecnologias de interfaces gestuais de forma a serem utilizados por pessoas com Dispraxia, criando mais potencialidades na alfabetização, caligrafia e letramento escolar com ambientes a distância de ensino aprendizagem.

Desta forma, recomenda-se a criação de guidelines que norteiem como devem ser realizadas as adaptações na arquitetura do Moodle para alterar o módulo de interface, facilitando a acessibilidade para pessoas com TDC nos AVAs e permitindo que tecnologias de interfaces gestuais sejam utilizadas com maior segurança.

O AVA a ser adaptado será o Moodle, já que é um sistema desenvolvido em PHP, baseado em código livre e estável, tendo sido projetado em princípios pedagógicos, além de ser um framework modularizado e com arquitetura focada no ambiente de um curso, facilitando a configuração, criação de guidelines e adaptação para reconhecimento apropriado pelas interfaces gestuais.

A tecnologia de interface gestual a ser utilizada será o Leapmotion, pois, em relação aos exemplos remetidos na Seção 2, é um dispositivo tecnológico com uma calibração apropriada aos sujeitos com TDC e com um sistema focado somente nos gestos e movimentos das mãos, facilitando o manuseio e utilização da ferramenta. Além disso, ele possui baixo custo de aquisição em relação aos concorrentes, uma dimensão física apropriada para locomoção de dispráxicos e um sensor com capacidade de deteç̧ão mais preciso que os concorrentes.

As recomendações para as adaptações criarão subsídios para melhorar a acessibilidade no Moodle ao usarem interfaces gestuais e tecnologias que se utilizam delas, gerando outras potencialidades de aprendizagem, além de tornar mais intuitivo o acesso ao Moodle para dispráxicos e indivíduos com outros transtornos.

\section{TRABALHOS EXISTENTES}

Alguns estudos destacam os Ambientes Virtuais de Aprendizagem, as interfaces touchless e o TDC, suas características e relevâncias. Poucos, entretanto, são os que destacam estas três temáticas juntas e a integração entre AVAs e interfaces gestuais.

Sobre AVAs e a utilização com interfaces sensíveis ao toque ou touchscreen, têm-se os trabalhos de [Oliveira et al. 2014] e [Tang et al. 2014].

Sobre a Dispraxia, os trabalhos de [Othman and KeayBright 2010] e [Othman and Keay-Bright 2011] usam técnicas de animação para o desenvolvimento de habilidades de caligrafia, assim como o estudo clássico de [Polatajko and Cantin 2005], que faz uma revisão sobre as intervenções realizadas em pessoas com TDC e sobre pesquisa realizadas até então.

Já estudos que abordam as interfaces gestuais, podese citar o [Erazo and Pico 2014] como modelo de revisão bibliográfica recente sobre touchless e as relevantes pesquisas de [Zhao et al. 2014], [Chen et al. 2014] e [Placitelli and Gallo 2012], além dos [Bartoli et al. 2014] e [Garzotto et al. 2014].

Estes trabalhos, portanto, servem de base para esta proposta de investigação, destacando a relevância das interfaces gestuais para pessoas com Dispraxia ao utilizarem AVAs através do Leapmotion.

\section{CONSIDERAÇÕES FINAIS}

Esta pesquisa ressalta a importância e propõe a adaptação de Ambientes Virtuais de Aprendizagem para as tecnologias de interfaces gestuais de forma a serem utilizados por pessoas com Dispraxia. 


\section{. . F.'Ingenierías}

Com essa proposta de adequação, dispráxicos podem utilizar tecnologias touchless para acessar conteúdos nesses ambientes de ensino de forma mais natural, pois estas interfaces são mais intuitivas que as outras e promovem uma melhor interação humanocomputador, adaptando-se às suas realidades e proporcionando uma potencial melhoria na qualidade de vida desses usuários.

Como resultado desta fase de projeto, espera-se criar guidelines que norteiem como devem ser realizadas as adaptações na arquitetura do Moodle para mudar o módulo de interface, facilitando a acessibilidade para pessoas com TDC nos AVAs e permitindo que tecnologias de interfaces gestuais como o Leapmotion sejam utilizadas com maior segurança. Além disso, espera-se que estes ambientes possam ser acessados por mais dispráxicos e pessoas com outros distúrbios.

Como etapa seguinte, este trabalho testará a tecnologia de interface virtual Leapmotion em um AVA por indivíduos com Dispraxia, analisando-se seus comportamentos durante a interação e o aprimorando dessas guidelines.

\section{AGRADECIMENTOS}

Os autores agradecem a Universidade Federal do Piauí (UFPI) e ao Fundo Mackenzie de Pesquisa da Universidade Presbiteriana Mackenzie (UPM).

\section{REFERÊNCIAS}

Antonio, N. S., Levy, P. C., Caetano, R., and Souza, P. G. (2014). Design of a brazilian portuguese virtual keyboard for people with severe motor disability. In Proceedings of the 13th Brazilian Symposium on Human Factors in Computing Systems, pages 14-20. Sociedade Brasileira de Computação

Bartoli, L., Garzotto, F., Gelsomini, M., Oliveto, L., and Valoriani, M. (2014). Designing and evaluating touchless playful interaction for asd children. In Proceedings of the 2014 conference on Interaction design and children, pages 17-26. ACM.

Cardoso, A. M. P., de Carvalho, R. C., da Silva, S. d. A. A., Pio, D. C., da Silveira, P. H. B. R., et al. (2014). Facil: Modelo para avaliação da literacia digital e informacional. Revista Brasileira de Informática na Educação, 22(03):46.

Chen, X., Schwarz, J., Harrison, C., Mankoff, J., and Hudson, S. E. (2014). Air+ touch: interweaving touch \& in-air gestures. In Proceedings of the 27th annual ACM symposium on User interface software and technology, pages 519-525. ACM.

da Costa Ferro, M. R., Paraguaçu, F., Peres, A. L., and Marinho, M. F. (2014). Recomendação assistida por computador de materiais didáticos em ambientes virtuais de aprendizagem. Informática na educação: teoria \& prática, 17(1).

da Silva, R. R. T., de Lima, R. W., Leite, C. R. M., and da Silva, R. R. T. (2014). Investigação de segurança no moodle. RENOTE - Revista Novas Tecnologias na Educação, 12(2).

Erazo, O. and Pico, R. (2014). Interfaces de usuario basadas en gestos manuales sin contacto para la sala de clases: una revision bibliográfica. Enfoque UTE, 5(4):pp-34. 


\section{Ingenierías: $:$ :}

Erazo, O. and Pino, J. (2014). Estimating the difficulty of touchless hand gestures. Latin America Transactions, IEEE (Revista IEEE America Latina), 12(1):17-22.

Garzotto, F., Valoriani, M., and Bartoli, L. (2014). Touchless motion-based interaction for therapy 